\title{
Transmission Model of Chikungunya Fever in the Presence of Two Species of Aedes Mosquitoes
}

\author{
${ }^{1}$ Surapol Naowarat and ${ }^{2}$ I. Ming Tang \\ ${ }^{1}$ Department of Mathematics, Faculty of Science and Technology, \\ Suratthani Rajabhat University, Surat Thani, 84100, Thailand \\ ${ }^{2}$ Department of Mathematics, Faculty of Science, \\ Silpakorn University, Nakorn Pathom 73000, Thailand
}

Received 2012-08-07, Revised 2013-04-17; Accepted 2013-05-27

\begin{abstract}
In 2008 there was a large outbreak of Chikungunya fever in the south of Thailand. Chikungunya fever is a febrile disease transmitted to humans by the bite of infected Aedes mosquitoes. The symptoms of this disease are a sudden onset of a fever, chills, headache, nausea, vomiting, joint pain with or without swelling, low back pain and rash. In this study we study the effects of there being two species of Aedes mosquito (Aedes aegypti and Aedes albopictus) present. In this study, we assume that both the human and mosquito populations are constant. A dynamical model of Chikungunya fever is proposed and analyzed. The RouthHurwitz criteria are used to determine the stability of the model. The conditions which would lead to either the disease free equilibrium state or the disease endemic equilibrium state to exist is determined. The numerical simulations are done in order to illustrate the behaviors of transmission of disease for different values of parameters. It is shown that the destruction of breeding sites could be an effective method to control this disease.
\end{abstract}

Keywords: Mathematical Model, Chikungunya Fever, Basic Reproductive Number, Equilibrium Point, Aedes Mosquitoes

\section{INTRODUCTION}

Chikungunya fever is an emerging, mosquito-borne disease caused by an alphavirus of Togaviridae family, which first was isolated in 1953 in Tanzania (PAHO, 2011). The symptoms of this disease are a high fever, extreme joints paints and an irritating skin rash (Tilston et al., 2009). This disease is self-limiting and some patients suffered from along-lasting arthritis akin (Kucharz and Cebula-Byrska, 2012). In the major outbreak of this disease in 2005 on the island of $\mathrm{R} \overline{\mathrm{e}}$ union, 244,000 out of a population of 775,000 inhabitants reported that they had experienced these symptoms (Moulay et al., 2011). Chikungunya Virus (CHIKV) is transmitted to humans by the bite of an infected Aedes mosquito, widespread in some tropical regions. The Ae. albopictus mosquito is a highly competent vector for this virus (Poletti et al., 2011). A genetic change at position 226 in the gene for the glycolprotein E1/E2 created a mutated Chikungunya virus strain which had an increased capability for replication in the Ae. albopictus mosquito (Tsetsarkin, 2009).

Mathematical modeling is a useful tool for understanding and describing the transmission of this disease. Pongsumpun (2010) has studied the effects of there being a seasonal variation in the number of mosquitoes on the transmission of this disease. The results showed that length of the outbreak would be shorter if the basic reproduction number would be higher. Poletti et al. (2011) developed a model describing the temporal dynamics of the vector depending on climate factors, coupled to an epidemic transmission model describing the

Corresponding Author: Surapol Naowarat, Department of Mathematics, Faculty of Science and Technology, Suratthani Rajabhat University, Surat Thani, 84100, Thailand 
spread of the epidemic in both humans and mosquitoes. The cumulative number of notified cases predicted by the model was 185 on average, in good agreement with observed data. They found that the basic reproduction number was in the range of 1.8-6. Yakob and Clements (2013) proposed a simple model of the virus transmission between humans and mosquitoes. This model is fitted with data from Reunion epidemic, with basic reproductive number is 4.1 . In a latter study, Moulay and Pigne (2013) proposed a metapopulation model to represent both a high-resolution humans and mosquitoes. Numerical results showed the impact of the geographical environment and populations' mobility on the spread of the disease.

In Asia and the Indian Ocean region the main CHIKV vectors are the Ae. aegypti and Ae. albopictus mosquitoes. Ae. albopictus is particularly resilient and it can survive in both rural and urban area. It has shown a remarkable capacity to adapt to human being and to urbanization, allowing the Ae. albopictus mosquito to supersede Ae. aegypti mosquito. Ae. albopictus has a relatively longer life expectancy about 4 to 8 weeks and a flight radius of $400-600 \mathrm{~m}$. It is aggressive biter and is diurnal. Preechaporn et al. (2007) investigated the seasonal prevalence of Ae. aegypti and Ae. albopictus in three topographical areas (mangrove, rice paddy and mountainous areas) in both wet and dry seasons in Nakhon Si Thammarat province, Thailand. Ae. aegypti larval indices were higher than the Ae. Albopictus larval indices in all three topographical areas during both seasons. In this study, we have formulated and analyze the Chikungunya fever model in which both species of the aedes mosquitoes are present. In most countries, only one species are present. In Thailand however both species are present.

\section{MATERIALS AND METHODS}

\subsection{Model Formulation}

In our model, we assume that human population and mosquito population are constant denoted by $\mathrm{N}_{h}$ and $\mathrm{N}_{\mathrm{m}}$ respectively. The dynamics of the disease is depicted in the compartment diagram, Fig. 1.

The human population is divided into the susceptible human $\left(\overline{\mathrm{S}}_{\mathrm{h}}\right)$, the infected human $\left(\overline{\mathrm{I}}_{\mathrm{h}}\right)$ and the recover human population $\left(\overline{\mathrm{R}}_{\mathrm{h}}\right)$ compartment. The mosquito population is divided into four compartments, the susceptible Ae aegypti mosquito $\left(\overline{\mathrm{S}}_{\mathrm{m} 1}\right)$, the infected $A e$. aegypti mosquito $\left(\overline{\mathrm{I}}_{\mathrm{m} 1}\right)$, the susceptible Ae. albopictus mosquito $\left(\overline{\mathrm{S}}_{\mathrm{m} 2}\right)$ and the infected Ae. albopictus mosquito $\left(\overline{\mathrm{I}}_{\mathrm{m} 2}\right)$ compartment, the recovered compartments for the mosquito does not exist, since the mosquitoes do not recover after they are infected for over all their life.

The transmission dynamics of the Chikungunya fever are described by the following ordinary differential equations:

$$
\begin{aligned}
& \frac{\mathrm{d} \overline{\mathrm{S}}_{\mathrm{h}}}{\mathrm{dt}}=\lambda_{\mathrm{h}} \mathrm{N}_{\mathrm{h}}-\left(\gamma_{\mathrm{m} 1} \overline{\mathrm{I}}_{\mathrm{m} 1}+\gamma_{\mathrm{m} 2} \overline{\mathrm{I}}_{\mathrm{m} 2}\right) \overline{\mathrm{S}}_{\mathrm{h}}-\mu_{\mathrm{h}} \overline{\mathrm{S}}_{\mathrm{h}} \\
& \frac{\mathrm{d} \overline{\mathrm{I}}_{\mathrm{h}}}{\mathrm{dt}}=\left(\gamma_{\mathrm{m} 1} \overline{\mathrm{I}}_{\mathrm{m} 1}+\gamma_{\mathrm{m} 2} \overline{\mathrm{I}}_{\mathrm{m} 2}\right) \overline{\mathrm{S}}_{\mathrm{h}}-\left(\mathrm{r}_{\mathrm{h}}+\mu_{\mathrm{h}}\right) \overline{\mathrm{I}}_{\mathrm{h}} \\
& \frac{\mathrm{d} \overline{\mathrm{R}}_{\mathrm{h}}}{\mathrm{dt}}=\mathrm{r}_{\mathrm{h}} \overline{\mathrm{I}}_{\mathrm{h}}-\mu_{\mathrm{h}} \overline{\mathrm{R}}_{\mathrm{h}} \\
& \frac{\mathrm{d} \overline{\mathrm{S}}_{\mathrm{m} 1}}{\mathrm{dt}}=\mathrm{A}_{1}-\gamma_{\mathrm{h}} \overline{\mathrm{I}}_{\mathrm{h}} \overline{\mathrm{S}}_{\mathrm{m} 1}-\mu_{\mathrm{m} 1} \overline{\mathrm{S}}_{\mathrm{m} 1} \\
& \frac{\mathrm{d} \overline{\mathrm{I}}_{\mathrm{m} 1}}{\mathrm{dt}}=\gamma_{\mathrm{h}} \overline{\mathrm{I}}_{\mathrm{h}} \overline{\mathrm{S}}_{\mathrm{m} 1}-\mu_{\mathrm{m} 1} \overline{\mathrm{I}}_{\mathrm{m} 1} \\
& \frac{\mathrm{d} \overline{\mathrm{S}}_{\mathrm{m} 2}}{\mathrm{dt}}=\mathrm{A}_{2}-\gamma_{\mathrm{h}} \overline{\mathrm{I}}_{\mathrm{h}} \overline{\mathrm{S}}_{\mathrm{m} 2}-\mu_{\mathrm{m} 2} \overline{\mathrm{S}}_{\mathrm{m} 2} \\
& \frac{\mathrm{d} \overline{\mathrm{I}}_{\mathrm{m} 2}}{\mathrm{dt}}=\gamma_{\mathrm{h}} \overline{\mathrm{I}}_{\mathrm{h}} \overline{\mathrm{S}}_{\mathrm{m} 2}-\mu_{\mathrm{m} 2} \overline{\mathrm{I}}_{\mathrm{m} 2}
\end{aligned}
$$

The Equation (1c, 1d and 1f) are dropped since it will be assumed that the human and mosquito population are constant. i.e., $\quad \overline{\mathrm{R}}_{\mathrm{h}}=\mathrm{N}_{\mathrm{h}}-\overline{\mathrm{S}}_{\mathrm{h}}-\overline{\mathrm{I}}_{\mathrm{h}}, \overline{\mathrm{S}}_{\mathrm{m} 1}=\mathrm{N}_{\mathrm{m} 1}-\overline{\mathrm{I}}_{\mathrm{ml}} \quad$ and $\overline{\mathrm{S}}_{\mathrm{m} 2}=\mathrm{N}_{\mathrm{m} 2}-\overline{\mathrm{I}}_{\mathrm{m} 2}$. The number of dependent variables reduced to four, which we pick to be $\mathrm{S}_{\mathrm{h}}, \mathrm{I}_{\mathrm{h}}, \mathrm{I}_{\mathrm{m} 1}, \mathrm{I}_{\mathrm{m} 2}$.

To analyze the model by normalizing the Equation (1a-1g) and defining new variables Equation 2a-2d:

$$
\begin{aligned}
& \mathrm{S}_{\mathrm{h}}=\frac{\overline{\mathrm{S}}_{\mathrm{h}}}{\mathrm{N}_{\mathrm{h}}}, \mathrm{I}_{\mathrm{h}}=\frac{\overline{\mathrm{I}}_{\mathrm{h}}}{\mathrm{N}_{\mathrm{h}}}, \mathrm{R}_{\mathrm{h}}=\frac{\overline{\mathrm{R}}_{\mathrm{h}}}{\mathrm{N}_{\mathrm{h}}} \\
& \mathrm{S}_{\mathrm{m} 1}=\frac{\overline{\mathrm{S}}_{\mathrm{m} 1}}{\mathrm{~N}_{\mathrm{m} 1}}=\frac{\overline{\mathrm{S}}_{\mathrm{m} 1}}{\mathrm{~A}_{1} /\left(\mu_{\mathrm{m} 1}\right)}, \mathrm{I}_{\mathrm{m} 1}=\frac{\overline{\mathrm{I}}_{\mathrm{m} 1}}{\mathrm{~N}_{\mathrm{m} 1}}=\frac{\overline{\mathrm{I}}_{\mathrm{m} 1}}{\mathrm{~A}_{1} /\left(\mu_{\mathrm{m} 1}\right)} \\
& \mathrm{S}_{\mathrm{m} 2}=\frac{\overline{\mathrm{S}}_{\mathrm{m} 2}}{\mathrm{~N}_{\mathrm{m} 2}}=\frac{\overline{\mathrm{S}}_{\mathrm{m} 2}}{\mathrm{~A}_{2} /\left(\mu_{\mathrm{m} 2}\right)}, \mathrm{I}_{\mathrm{m} 2}=\frac{\overline{\mathrm{I}}_{\mathrm{m} 2}}{\mathrm{~N}_{\mathrm{m} 2}}=\frac{\overline{\mathrm{I}}_{\mathrm{m} 2}}{\mathrm{~A}_{2} /\left(\mu_{\mathrm{m} 2}\right)}
\end{aligned}
$$

Since the total human and mosquito populations are constant, thus the time rate of change of human population equal to zero, i.e., $\frac{d \bar{S}_{h}}{d t}+\frac{d \bar{I}_{h}}{d t}+\frac{d \bar{R}_{h}}{d t}=0$. 


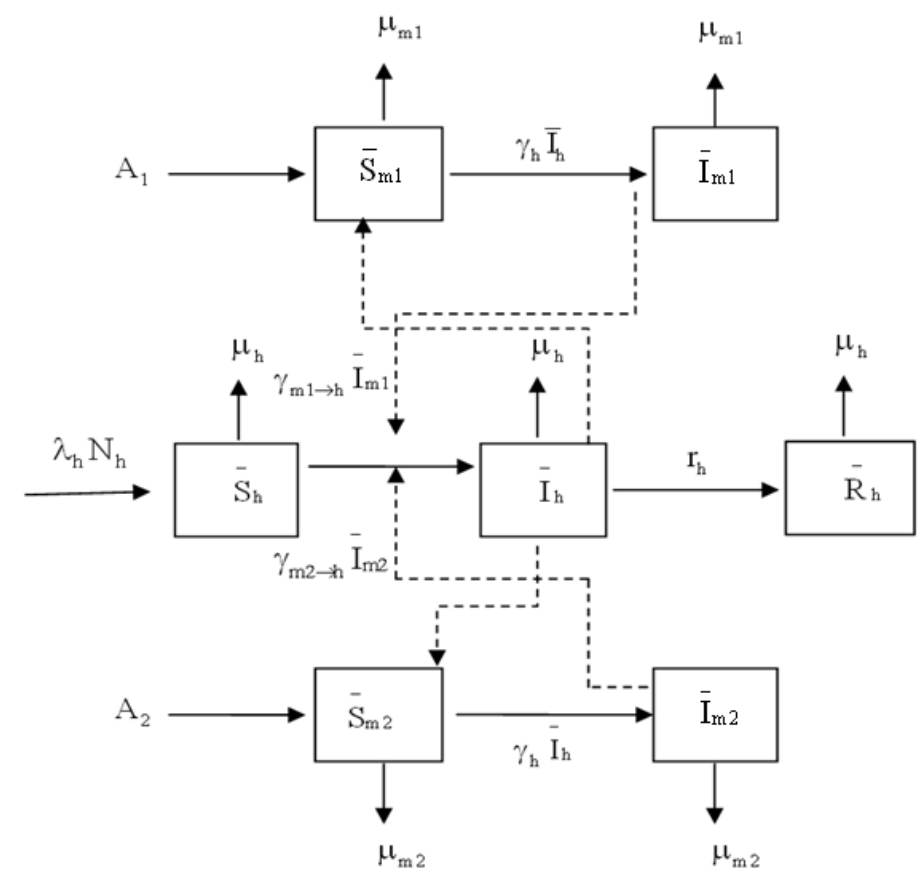

Fig. 1. Diagram for the dynamical transmission of Chikungunya fever

It means that the birth rate and the death rate of human population are equal, that is $\lambda_{\mathrm{h}}=\mu_{\mathrm{h}}$. The total number of Ae. aegypti mosquito and Ae. albopictus mosquito at equilibrium equal to $\frac{A_{1}}{\mu_{\mathrm{m} 1}}$ and $\frac{A_{2}}{\mu_{\mathrm{m} 2}}$, respectively.

where, $\mathrm{N}_{\mathrm{h}}$ is the total human population:

- $\quad \mathrm{N}_{\mathrm{m} 1}\left(\mathrm{~N}_{\mathrm{m} 2}\right)$ is the total Ae. aegypti (Ae. albopictus) mosquito population

- $\mathrm{S}_{\mathrm{h}}, \mathrm{I}_{\mathrm{h}}, \mathrm{R}_{\mathrm{h}}$ is the number of susceptible, infected, recovered human population, respectively

- $\mathrm{S}_{\mathrm{m} 1},\left(\mathrm{I}_{\mathrm{m} 1}\right)$ is the number of susceptible (infected) $A e$. aegypti mosquito population

- $\mathrm{S}_{\mathrm{m} 2}\left(\mathrm{I}_{\mathrm{m} 2}\right)$ is the number of susceptible (infected) Ae. albopictus mosquito population

- $\quad \lambda_{\mathrm{h}}\left(\mu_{\mathrm{h}}\right)$ is the birth (death) rate of human population

- $\mathrm{A}_{1}\left(\mathrm{~A}_{2}\right)$ is the recruit rate of Ae. aegypti (Ae. albopictus ) mosquito population

- $\gamma_{\mathrm{m} 1 \rightarrow \mathrm{h}}\left(\gamma_{\mathrm{m} 2 \rightarrow \mathrm{h}}\right)$ is the transmission rate of CHIKV from infected Ae. aegypti (Ae. albopictus) mosquito to human population

- $\mathrm{r}_{\mathrm{h}}$ is the recovery rate of human population

- $\quad b$ is the biting rate of mosquito population

- $\beta_{\mathrm{h} \rightarrow \mathrm{m} 1}\left(\beta_{\mathrm{h} \rightarrow \mathrm{m} 2}\right)$ is the probability that CHIV will be transmitted from infected human population to $A e$. aegypti (Ae. albopictus) mosquito population
- $\gamma_{\mathrm{h} \rightarrow \mathrm{m} 1}$ is the transmission rate of CHIKV from infected human population to susceptible Ae. aegypti mosquito population, i.e.:

$$
\gamma_{\mathrm{h} \rightarrow \mathrm{ml}}=\frac{\mathrm{b} \beta_{\mathrm{h} \rightarrow \mathrm{ml}}}{\mathrm{N}_{\mathrm{m}}}
$$

$\gamma_{\mathrm{h} \rightarrow \mathrm{m} 2}$ is the transmission rate of CHIKV from infected human population to susceptible Ae. albopictus mosquito population, i.e.:

$\gamma_{\mathrm{h} \rightarrow \mathrm{m} 2}=\frac{\mathrm{b} \beta_{\mathrm{h} \rightarrow \mathrm{m} 2}}{\mathrm{~N}_{\mathrm{m}}}$

We assume that the transmission rate of CHIV from infected human population to both mosquitoes are equal, $\gamma_{\mathrm{h} \rightarrow \mathrm{m} 1}=\gamma_{\mathrm{h} \rightarrow \mathrm{m} 2}=\gamma_{\mathrm{h}}$ and:

$\mathrm{N}_{\mathrm{m}}=\mathrm{N}_{\mathrm{m} 1}+\mathrm{N}_{\mathrm{m} 2}$

$\mu_{\mathrm{m} 1}\left(\mu_{\mathrm{m} 2}\right)$ is the death rate of Ae. aegypti (Ae.albopictus) mosquito population.

The reduced model is depicted as following:

$$
\begin{aligned}
\frac{d S_{h}}{d t}= & \mu_{\mathrm{h}}\left(1-S_{\mathrm{h}}\right)-\gamma_{\mathrm{m} 1 \rightarrow \mathrm{h}}\left(\mathrm{A}_{1} / \mu_{\mathrm{m} 1}\right) \mathrm{I}_{\mathrm{m} 1} \mathrm{~S}_{\mathrm{h}} \\
& -\gamma_{\mathrm{m} 2 \rightarrow \mathrm{h}}\left(\mathrm{A}_{2} / \mu_{\mathrm{m} 2}\right) \mathrm{I}_{\mathrm{m} 2} \mathrm{~S}_{\mathrm{h}}
\end{aligned}
$$




$$
\begin{aligned}
& \frac{\mathrm{dI}_{\mathrm{h}}}{\mathrm{dt}}=\gamma_{\mathrm{m} 1 \rightarrow \mathrm{h}}\left(\mathrm{A}_{1} / \mu_{\mathrm{m} 1}\right) \mathrm{I}_{\mathrm{m} 1} \mathrm{~S}_{\mathrm{h}} \\
& +\gamma_{\mathrm{m} 2 \rightarrow \mathrm{h}}\left(\mathrm{A}_{2} / \mu_{\mathrm{m} 2}\right) \mathrm{I}_{\mathrm{m} 2} \mathrm{~S}_{\mathrm{h}}-\left(\mathrm{r}_{\mathrm{h}}+\mu_{\mathrm{h}}\right) \mathrm{I}_{\mathrm{h}} \\
& \frac{d I_{m 1}}{d t}=\gamma_{h} N_{h} I_{h}\left(1-I_{m 1}\right)-\mu_{m 1} I_{m 1} \\
& \frac{d I_{m 2}}{d t}=\gamma_{h} N_{h} I_{h}\left(1-I_{m 2}\right)-\mu_{m 2} I_{m 2}
\end{aligned}
$$

\subsection{Analysis of the Model}

\subsubsection{Equilibrium Points}

We first determine the equilibrium points and investigate their stability. It is found that the system has two possible equilibrium points: the disease free equilibrium point and an endemic equilibrium point. Two equilibrium points are found by setting the RHS of Equation 3-6 to zero. Doing this, we obtained.

\subsection{Disease Free Equilibrium Point $\left(\mathbf{E}_{0}\right)$}

In the absence of disease, that is $\mathrm{I}_{\mathrm{h}}=0, \mathrm{I}_{\mathrm{m} 1}=0, \mathrm{I}_{\mathrm{m} 2}=$ 0. Equation 3 reduces to:

$$
\frac{\mathrm{dS}_{\mathrm{h}}}{\mathrm{dt}}=\mu_{\mathrm{h}}\left(1-\mathrm{S}_{\mathrm{h}}\right)
$$

The solution of this equation is $\mathrm{S}_{\mathrm{h}}=1$. The disease free state becomes $\mathrm{E}_{0}=(1,0,0,0)$.

\subsection{Endemic Equilibrium Point $\left(E_{1}\right)$}

In the case where the disease is presented, we will have $\mathrm{I}_{\mathrm{h}} \neq 0, \mathrm{I}_{\mathrm{m} 1} \neq 0, \mathrm{I}_{\mathrm{m} 2} \neq 0$, This gives:

$$
\begin{aligned}
& I_{h}^{*}=\frac{M_{2} P_{1}+M_{1} P_{2}}{P_{1}+P_{2}} \\
& I_{m 1}^{*}=\frac{M_{1} P_{2}+M_{2} P_{1}}{M_{1} P_{2}+M_{2} P_{1}+M_{1}\left(P_{1}+P_{2}\right)} \\
& I_{m 2}^{*}=\frac{M_{1} P_{2}+M_{2} P_{1}}{M_{1} P_{2}+M_{2} P_{1}+M_{2}\left(P_{1}+P_{2}\right)} \\
& S_{h}^{*}=\frac{M_{11} M_{22}}{N_{11} M_{22}+N_{22} M_{11}+M_{11} M_{22}}
\end{aligned}
$$

With:

$$
\begin{aligned}
& \mathrm{P}_{1}=\frac{\gamma_{\mathrm{m} 1 \rightarrow \mathrm{h}} \mathrm{A}_{1}}{\mu_{\mathrm{h}} \mu_{\mathrm{m} 1}}, \mathrm{P}_{2}=\frac{\gamma_{\mathrm{m} 2 \rightarrow \mathrm{h}} \mathrm{A}_{2}}{\mu_{\mathrm{h}} \mu_{\mathrm{m} 2}} \\
& \mathrm{M}_{1}=\frac{\mu_{\mathrm{m} 1 \rightarrow \mathrm{h}}}{\gamma_{\mathrm{h}} \mathrm{N}_{\mathrm{h}}}, \mathrm{M}_{2}=\frac{\mu_{\mathrm{m} 2 \rightarrow \mathrm{h}}}{\gamma_{\mathrm{h}} \mathrm{N}_{\mathrm{h}}}, \mathrm{M}_{11}=\mathrm{M}_{1} \mathrm{P}_{2}+\mathrm{M}_{2} \mathrm{P}_{1}+\mathrm{M}_{1}\left(\mathrm{P}_{1}+\mathrm{P}_{2}\right) \\
& \mathrm{M}_{22}=\mathrm{M}_{1} \mathrm{P}_{2}+\mathrm{M}_{2} \mathrm{P}_{1}+\mathrm{M}_{2}\left(\mathrm{P}_{1}+\mathrm{P}_{2}\right), \mathrm{N}_{11}=\mathrm{P}_{1}\left(\mathrm{M}_{1} \mathrm{P}_{2}+\mathrm{M}_{2} \mathrm{P}_{1}\right)
\end{aligned}
$$

And:

$$
\mathrm{N}_{22}=\mathrm{P}_{2}\left(\mathrm{M}_{1} \mathrm{P}_{2}+\mathrm{M}_{2} \mathrm{P}_{1}\right)
$$

The

becomes $\mathrm{E}_{1}\left(\mathrm{~S}_{\mathrm{h}}^{*}, \mathrm{I}_{\mathrm{h}}^{*}, \mathrm{I}_{\mathrm{m} 1}^{*}, \mathrm{I}_{\mathrm{m} 2}^{*}\right)$.

\subsection{Basic Reproductive Number}

The basic reproductive number is obtained by the next generation method. In the notations of Driessche and Watmough (2002), we start with:

$$
\frac{\mathrm{dX}}{\mathrm{dt}}=\mathrm{F}_{\mathrm{i}}-\mathrm{v}_{\mathrm{i}}
$$

where, $F_{i}$ is the matrix of new infectious and $v_{i}$ is the matrix for the transfers between the compartments in the infective equations. Specifically, we have:

$$
X=\left[\begin{array}{l}
S_{h} \\
I_{h} \\
I_{m 1} \\
I_{m 2}
\end{array}\right], F_{i}=\left[\begin{array}{c}
0 \\
\frac{\gamma_{m 1 \rightarrow h}\left(A_{1} / \mu_{m 1}\right) S_{h} I_{m 1}}{N_{h}}+\frac{\gamma_{m 2 \rightarrow h}\left(A_{2} / \mu_{m 2}\right) S_{h} I_{m 2}}{N_{h}} \\
0
\end{array}\right]
$$

And:

$$
V_{i}=\left[\begin{array}{c}
-\mu_{h}+\gamma_{m 1 \rightarrow h}\left(A_{1} / \mu_{m 1}\right) S_{h} I_{m 1}+\gamma_{m 2 \rightarrow h}\left(A_{2} / \mu_{m 2}\right) S_{h} I_{m 2}+\mu_{h} S_{h} \\
\left(r_{h}+\mu_{h}\right) I_{h} \\
-\gamma_{h} N_{h} I_{h}+\gamma_{h} N_{h} I_{h} I_{m 1}+\mu_{m 1} I_{m 1} \\
-\gamma_{h} N_{h} I_{h}+\gamma_{h} N_{h} I_{h} I_{m 2}+\mu_{m 2} I_{m 2}
\end{array}\right]
$$

where, $F=\left[\frac{\partial F_{i}\left(E_{0}\right)}{\partial X_{j}}\right]$ and $V=\left[\frac{\partial V_{i}\left(E_{0}\right)}{\partial X_{j}}\right]$ for all $i, j=1,2$, 3,4 are the Jacobian matrix of $F$ and $v$ at $E_{0}$. The basic reproductive number, $\mathrm{R}_{0}$, is the threshold for the stability of the disease free equilibrium $\mathrm{E}_{0}$. It can be calculated by noting that:

$$
\mathrm{R}_{0}=\rho\left(\mathrm{FV}^{-1}\right)
$$

where, $\mathrm{FV}^{-1}$ is called the next generation matrix and $\rho\left(\mathrm{FV}^{-1}\right)$ is the spectral radius (dominant eigenvalue) of the matrix $\mathrm{FV}^{-1}$. For our model, the Jacobian matrices are:

$$
\mathrm{F}\left(\mathrm{E}_{0}\right)=\left[\begin{array}{cccc}
0 & 0 & 0 & 0 \\
0 & 0 & \gamma_{\mathrm{m} 1 \rightarrow \mathrm{h}}\left(\mathrm{A}_{1} / \mu_{\mathrm{m} 1}\right) & \gamma_{\mathrm{m} 2 \rightarrow \mathrm{h}}\left(\mathrm{A}_{2} / \mu_{\mathrm{m} 2}\right) \\
0 & 0 & 0 & 0 \\
0 & 0 & 0 & 0
\end{array}\right]
$$


And:

$$
\mathrm{V}\left(\mathrm{E}_{0}\right)=\left[\begin{array}{cccc}
\mu_{\mathrm{h}} & 0 & \gamma_{\mathrm{m} 1 \rightarrow \mathrm{h}}\left(\mathrm{A}_{1} / \mu_{\mathrm{m} 1}\right) & \gamma_{\mathrm{m} 2 \rightarrow \mathrm{h}}\left(\mathrm{A}_{2} / \mu_{\mathrm{m} 2}\right) \\
0 & \mathrm{r}_{\mathrm{h}}+\mu_{\mathrm{h}} & 0 & 0 \\
0 & -\gamma_{\mathrm{h}} \mathrm{N}_{\mathrm{h}} & \mu_{\mathrm{m} 1} & 0 \\
0 & -\gamma_{\mathrm{h}} \mathrm{N}_{\mathrm{h}} & 0 & \mu_{\mathrm{m} 2}
\end{array}\right]
$$

The inverse of $\mathrm{V}$ is:

$$
\mathrm{V}^{-1}\left(\mathrm{E}_{0}\right)=\left[\begin{array}{cccc}
\frac{1}{\mu_{\mathrm{h}}} & -\frac{\gamma_{\mathrm{h}} \mathrm{N}_{\mathrm{h}} \gamma_{\mathrm{m} 1 \rightarrow \mathrm{h}}\left(\mathrm{A}_{1} / \mu_{\mathrm{m} 1}\right)}{\mu_{\mathrm{h}}\left(\mathrm{r}_{\mathrm{h}}+\mu_{\mathrm{h}}\right) \mu_{\mathrm{m} 1}}-\frac{\gamma_{\mathrm{h}} \mathrm{N}_{\mathrm{h}} \gamma_{\mathrm{m} 2 \rightarrow \mathrm{h}}\left(\mathrm{A}_{2} / \mu_{\mathrm{m} 2}\right)}{\mu_{\mathrm{h}}\left(\mathrm{r}_{\mathrm{h}}+\mu_{\mathrm{h}}\right) \mu_{\mathrm{m} 2}} & \frac{\gamma_{\mathrm{m} 1 \rightarrow \mathrm{h}}\left(\mathrm{A}_{1} / \mu_{\mathrm{m} 1}\right)}{\mu_{\mathrm{h}} \mu_{\mathrm{m} 1}} & -\frac{\gamma_{\mathrm{m} 2 \rightarrow \mathrm{h}}\left(\mathrm{A}_{2} / \mu_{\mathrm{m} 2}\right)}{\mu_{\mathrm{h}} \mu_{\mathrm{m} 2}} \\
0 & \frac{1}{\mathrm{r}_{\mathrm{h}}+\mu_{\mathrm{h}}} & 0 & 0 \\
0 & \frac{\gamma_{\mathrm{h}} \mathrm{N}_{\mathrm{h}}}{\mu_{\mathrm{m} 1}\left(\mathrm{r}_{\mathrm{h}}+\mu_{\mathrm{h}}\right)} & \frac{1}{\mu_{\mathrm{m} 1}} & 0 \\
0 & -\frac{\gamma_{\mathrm{h}} \mathrm{N}_{\mathrm{h}}}{\mu_{\mathrm{m} 2}\left(\mathrm{r}_{\mathrm{h}}+\mu_{\mathrm{h}}\right)} & 0 & \frac{1}{\mu_{\mathrm{m} 2}}
\end{array}\right]
$$

This leads to:

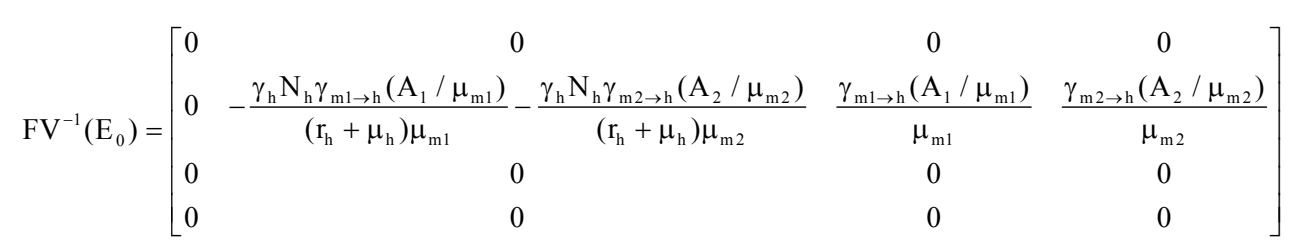

Thus:

$$
\rho\left(\mathrm{FV}^{-1}\left(\mathrm{E}_{0}\right)\right)=\frac{\gamma_{\mathrm{h}} \mathrm{N}_{\mathrm{h}} \gamma_{\mathrm{m} 1 \rightarrow \mathrm{h}}\left(\mathrm{A}_{1} / \mu_{\mathrm{m} 1}\right)}{\left(\mathrm{r}_{\mathrm{h}}+\mu_{\mathrm{h}}\right) \mu_{\mathrm{m} 1}}+\frac{\gamma_{\mathrm{h}} \mathrm{N}_{\mathrm{h}} \gamma_{\mathrm{m} 2 \rightarrow \mathrm{h}}\left(\mathrm{A}_{2} / \mu_{\mathrm{m} 2}\right)}{\left(\mathrm{r}_{\mathrm{h}}+\mu_{\mathrm{h}}\right) \mu_{\mathrm{m} 2}}
$$

And so:

$$
\mathrm{R}_{0}=\frac{\gamma_{\mathrm{h}} \mathrm{N}_{\mathrm{h}} \gamma_{\mathrm{m} 1 \rightarrow \mathrm{h}}\left(\mathrm{A}_{1} / \mu_{\mathrm{m} 1}\right)}{\left(\mathrm{r}_{\mathrm{h}}+\mu_{\mathrm{h}}\right) \mu_{\mathrm{m} 1}}+\frac{\gamma_{\mathrm{h}} \mathrm{N}_{\mathrm{h}} \gamma_{\mathrm{m} 2 \rightarrow \mathrm{h}}\left(\mathrm{A}_{2} / \mu_{\mathrm{m} 2}\right)}{\left(\mathrm{r}_{\mathrm{h}}+\mu_{\mathrm{h}}\right) \mu_{\mathrm{m} 2}}
$$

\subsection{Local Asymptotical Stability}

The local stability of an equilibrium point is determined from the Jacobian matrix of the system of ordinary differential Equation 3-6 evaluated at each equilibrium point. The Jacobian matrix at the disease free state $\mathrm{E}_{0}$ is Equation 11:

$\mathrm{J}_{0}=\left[\begin{array}{cccc}-\mu_{\mathrm{h}} & 0 & -\frac{\gamma_{\mathrm{m} 1 \rightarrow \mathrm{h}} \mathrm{A}_{1}}{\mu_{\mathrm{m} 1}} & -\frac{\gamma_{\mathrm{m} 2 \rightarrow \mathrm{h}} \mathrm{A}_{2}}{\mu_{\mathrm{m} 2}} \\ 0 & -\left(\mathrm{r}_{\mathrm{h}}+\mu_{\mathrm{h}}\right) & \frac{\gamma_{\mathrm{m} 1 \rightarrow \mathrm{h}} \mathrm{A}_{1}}{\mu_{\mathrm{m} 1}} & \frac{\gamma_{\mathrm{m} 2 \rightarrow \mathrm{h}} \mathrm{A}_{2}}{\mu_{\mathrm{m} 2}} \\ 0 & \gamma_{\mathrm{h}} \mathrm{N}_{\mathrm{h}} & -\mu_{\mathrm{m} 1} & 0 \\ 0 & \gamma_{\mathrm{h}} \mathrm{N}_{\mathrm{h}} & 0 & -\mu_{\mathrm{m} 2}\end{array}\right]$
The eigenvalues of the $\mathrm{J}_{0}$ are obtained by solving det $\left(\mathrm{J}_{0}-\lambda \mathrm{I}\right)=0$. We obtained the characteristic Equation 12:

$\left(\lambda+\mu_{\mathrm{h}}\right)\left(\lambda^{3}+\mathrm{a}_{1} \lambda^{2}+\mathrm{a}_{2} \lambda+\mathrm{a}_{3}\right)=0$

Where:

$$
\begin{aligned}
\mathrm{a}_{1}= & \mathrm{r}_{\mathrm{h}}+\mu_{\mathrm{h}}+\mu_{\mathrm{m} 1}+\mu_{\mathrm{m} 2}, \\
\mathrm{a}_{2}= & \left(\mathrm{r}_{\mathrm{h}}+\mu_{\mathrm{h}}\right)\left(\mu_{\mathrm{m} 1}+\mu_{\mathrm{m} 2}\right)+\mu_{\mathrm{m} 1} \mu_{\mathrm{m} 2}-\frac{\gamma_{\mathrm{h}} \mathrm{N}_{\mathrm{h}} \gamma_{\mathrm{m} 1 \rightarrow \mathrm{h}} \mathrm{A}_{1}}{\mu_{\mathrm{m} 1}} \\
& -\frac{\gamma_{\mathrm{h}} \mathrm{N}_{\mathrm{h}} \gamma_{\mathrm{m} 2 \rightarrow \mathrm{h}} \mathrm{A}_{2}}{\mu_{\mathrm{m} 2}}, \\
\mathrm{a}_{3}= & \left(\mathrm{r}_{\mathrm{h}}+\mu_{\mathrm{h}}\right)\left(\mu_{\mathrm{m} 1} \mu_{\mathrm{m} 2}\right)-\frac{\gamma_{\mathrm{h}} \mathrm{N}_{\mathrm{h}} \gamma_{\mathrm{m} 1 \rightarrow \mathrm{h}} \mathrm{A}_{1} \mu_{\mathrm{m} 2}}{\mu_{\mathrm{m} 1}} \\
& -\frac{\gamma_{\mathrm{h}} \mathrm{N}_{\mathrm{h}} \gamma_{\mathrm{m} 2 \rightarrow \mathrm{h}} \mathrm{A}_{2} \mu_{\mathrm{m} 1}}{\mu_{\mathrm{m} 2}}
\end{aligned}
$$

One of eigenvalues is $\lambda_{1}=-\mu_{\mathrm{h}}<0$. The other three eigenvalues are the solutions of the cubic equation $\lambda^{3}+a_{1} \lambda^{2}+a_{2} \lambda+a_{3}=0$. The roots of this equation are negative if the coefficients satisfied the three conditions of Routh-Hurwitz criteria (Allen, 2006). $\mathrm{E}_{0}$ will be locally asymptotically stable when the coefficients satisfy the conditions $a_{1}>0$ Equation 14: 
$\mathrm{a}_{3}>0$

$\mathrm{a}_{1} \mathrm{a}_{2}>\mathrm{a}_{3}$

Looking at the coefficients, Equation 13, we see that $\mathrm{a}_{1}$ is always positive and $\mathrm{a}_{3}$ will be positive when Equation 15:

$$
\frac{\gamma_{\mathrm{h}} \mathrm{N}_{\mathrm{h}} \gamma_{\mathrm{m} 1 \rightarrow \mathrm{h}} \mathrm{A}_{1} \mu_{\mathrm{m} 2}}{\mu_{\mathrm{m} 1}}+\frac{\gamma_{\mathrm{h}} \mathrm{N}_{\mathrm{h}} \gamma_{\mathrm{m} 2 \rightarrow \mathrm{h}} \mathrm{A}_{2} \mu_{\mathrm{m} 1}}{\mu_{\mathrm{m} 2}}<\left(\mathrm{r}_{\mathrm{h}}+\mu_{\mathrm{h}}\right)\left(\mu_{\mathrm{m} 1} \mu_{\mathrm{m} 2}\right)
$$

Thus, the disease free equilibrium point will be locally asymptotically stable when $\mathrm{R}_{\mathrm{o}}<1$.
Where Equation 16:

$$
\begin{aligned}
& \mathrm{R}_{\mathrm{o}}=\mathrm{R}_{01}+\mathrm{R}_{02} \\
& \mathrm{R}_{01}=\frac{\gamma_{\mathrm{h}} \mathrm{N}_{\mathrm{h}} \gamma_{\mathrm{m} 1 \rightarrow \mathrm{h}} \mathrm{A}_{1}}{\left(\mathrm{r}_{\mathrm{h}}+\mu_{\mathrm{h}}\right)\left(\mu_{\mathrm{m} 1} \mu_{\mathrm{m} 1}\right)}, \mathrm{R}_{02}=\frac{\gamma_{\mathrm{h}} \mathrm{N}_{\mathrm{h}} \gamma_{\mathrm{m} 2 \rightarrow \mathrm{h}} \mathrm{A}_{2}}{\left(\mathrm{r}_{\mathrm{h}}+\mu_{\mathrm{h}}\right)\left(\mu_{\mathrm{m} 2} \mu_{\mathrm{m} 2}\right)}
\end{aligned}
$$

\subsection{Disease Endemic Equilibrium Point}

To determine the stability of the endemic equilibrium point, $\mathrm{E}_{1}$, we first evaluate the Jacobian matrix at $E_{1}$ to get Equation 17:

$$
\mathrm{J}_{1}=\left[\begin{array}{ccccc}
-\mu_{\mathrm{h}}-\frac{\gamma_{\mathrm{m} 1 \rightarrow \mathrm{h}} \mathrm{A}_{1} \mathrm{I}_{\mathrm{m} 1}^{*}}{\mu_{\mathrm{m} 1}}-\frac{\gamma_{\mathrm{m} 2 \rightarrow \mathrm{h}} \mathrm{A}_{2} \mathrm{I}_{\mathrm{m} 2}^{*}}{\mu_{\mathrm{m} 2}} & 0 & -\frac{\gamma_{\mathrm{ml} \rightarrow \mathrm{h}} \mathrm{A}_{1} \mathrm{~S}_{\mathrm{h}}^{*}}{\mu_{\mathrm{m} 1}} & -\frac{\gamma_{\mathrm{m} 2 \rightarrow \mathrm{h}} \mathrm{A}_{2} \mathrm{~S}_{\mathrm{h}}^{*}}{\mu_{\mathrm{m} 2}} \\
\frac{\gamma_{\mathrm{ml} \rightarrow \mathrm{h}} \mathrm{A}_{1} \mathrm{I}_{\mathrm{m} 1}^{*}}{\mu_{\mathrm{m} 1}}+\frac{\gamma_{\mathrm{m} 2 \rightarrow \mathrm{h}} \mathrm{A}_{2} \mathrm{I}_{\mathrm{m} 2}^{*}}{\mu_{\mathrm{m} 2}} & -\left(\mathrm{r}+\mu_{\mathrm{h}}\right) & \frac{\gamma_{\mathrm{ml} \rightarrow \mathrm{h}} \mathrm{A}_{1} \mathrm{~S}_{\mathrm{h}}^{*}}{\mu_{\mathrm{m} 1}} & \frac{\gamma_{\mathrm{m} 2 \rightarrow h} \mathrm{~A}_{2} \mathrm{~S}_{\mathrm{h}}^{*}}{\mu_{\mathrm{m} 2}} \\
0 & & \gamma_{\mathrm{h}} \mathrm{N}_{\mathrm{h}}-\gamma_{\mathrm{h}} \mathrm{N}_{\mathrm{h}} \mathrm{I}_{\mathrm{m} 1}^{*} & -\gamma_{\mathrm{h}} \mathrm{N}_{\mathrm{h}} \mathrm{I}_{\mathrm{h}}^{*}-\mu_{\mathrm{m} 1} & 0 \\
0 & \gamma_{\mathrm{h}} \mathrm{N}_{\mathrm{h}}-\gamma_{\mathrm{h}} \mathrm{N}_{\mathrm{h}} \mathrm{I}_{\mathrm{m} 2}^{*} & 0 & -\gamma_{\mathrm{h}} \mathrm{N}_{\mathrm{h}} \mathrm{I}_{\mathrm{h}}^{*}-\mu_{\mathrm{m} 2}
\end{array}\right]
$$

where, $\mathrm{S}_{\mathrm{h}}^{*}, \mathrm{I}_{\mathrm{h}}^{*}, \mathrm{I}_{\mathrm{m} 1}^{*}, \mathrm{I}_{\mathrm{m} 2}^{*}$ are given by Equation 7-10. The characteristic equation of Jacobian matrix at $\mathrm{E}_{1}$, becomes Equation 18 :

$$
\lambda^{4}+b_{1} \lambda^{3}+b_{2} \lambda^{2}+b_{3} \lambda+b_{4}=0
$$

Where Equation 19:

$$
\begin{aligned}
& b_{1}=\mu_{m 1}+\mu_{m 2}+r_{h}+2 \mu_{h}+2 \gamma_{h} N_{h} I_{h}^{*}+\frac{\gamma_{m 1 \rightarrow h} A_{1} I_{m 1}^{*}}{\mu_{m 1}}+\frac{\gamma_{m 2 \rightarrow h} A_{2} I_{m 2}^{*}}{\mu_{m 2}}, \\
& \mathrm{~b}_{2}=\left(\mu_{\mathrm{m} 2}+\gamma_{\mathrm{h}} \mathrm{N}_{\mathrm{h}} \mathrm{I}_{\mathrm{h}}^{*}\right)\left(\mu_{\mathrm{m} 1}+\mathrm{r}_{\mathrm{h}}+\mu_{\mathrm{h}}+\gamma_{\mathrm{h}} \mathrm{N}_{\mathrm{h}} \mathrm{I}_{\mathrm{h}}^{*}\right)+\left(\mathrm{r}_{\mathrm{h}}+\mu_{\mathrm{h}}\right)\left(\mu_{\mathrm{m} 1}+\gamma_{\mathrm{h}} \mathrm{N}_{\mathrm{h}} \mathrm{I}_{\mathrm{h}}^{*}\right)-\left(\gamma_{\mathrm{h}} \mathrm{N}_{\mathrm{h}}\right)\left(1-\mathrm{I}_{\mathrm{m} 1}^{*}\right)\left(\frac{\gamma_{\mathrm{ml} \rightarrow \mathrm{h}} \mathrm{A}_{1}}{\mu_{\mathrm{m} 1}} \mathrm{~S}_{\mathrm{h}}^{*}\right) \\
& -\left(\gamma_{\mathrm{h}} \mathrm{N}_{\mathrm{h}}\right)\left(1-\mathrm{I}_{\mathrm{m} 2}^{*}\right)\left(\frac{\gamma_{\mathrm{m} 2 \rightarrow \mathrm{h}} \mathrm{A}_{2}}{\mu_{\mathrm{m} 2}} \mathrm{~S}_{\mathrm{h}}^{*}\right)+\left(\mu_{\mathrm{h}}+\frac{\gamma_{\mathrm{m} 1 \rightarrow \mathrm{h}} \mathrm{A}_{1} \mathrm{I}_{\mathrm{m} 1}^{*}}{\mu_{\mathrm{m} 1}}+\frac{\gamma_{\mathrm{m} 2 \rightarrow \mathrm{h}} \mathrm{A}_{2} \mathrm{I}_{\mathrm{m} 2}^{*}}{\mu_{\mathrm{m} 2}}\right)\left[\mu_{\mathrm{m} 1}+\mu_{\mathrm{m} 2}+\mathrm{r}_{\mathrm{h}}+\mu_{\mathrm{h}}+2 \gamma_{\mathrm{h}} \mathrm{N}_{\mathrm{h}} \mathrm{I}_{\mathrm{h}}^{*}\right] \\
& \mathrm{b}_{3}=\left(\mathrm{r}_{\mathrm{h}}+\mu_{\mathrm{h}}\right)\left(\mu_{\mathrm{m} 1}+\gamma_{\mathrm{h}} \mathrm{N}_{\mathrm{h}} \mathrm{I}_{\mathrm{h}}^{*}\right)\left(\mu_{\mathrm{m} 2}+\mathrm{r}_{\mathrm{h}} \mathrm{N}_{\mathrm{h}} \mathrm{I}_{\mathrm{h}}^{*}\right)-\left(\mu_{\mathrm{m} 1}+\gamma_{\mathrm{h}} \mathrm{N}_{\mathrm{h}} \mathrm{I}_{\mathrm{h}}^{*}\right)\left(\gamma_{\mathrm{h}} \mathrm{N}_{\mathrm{h}}\right)\left(1-\mathrm{I}_{\mathrm{m} 2}^{*}\right)\left(\frac{\gamma_{\mathrm{m} 2 \rightarrow \mathrm{h}} \mathrm{A}_{1} \mathrm{~S}_{\mathrm{h}}^{*}}{\mu_{\mathrm{m} 2}}\right) \\
& -\left(\mu_{\mathrm{m} 2}+\gamma_{\mathrm{h}} \mathrm{N}_{\mathrm{h}} \mathrm{I}_{\mathrm{h}}^{*}\right)\left(\gamma_{\mathrm{h}} \mathrm{N}_{\mathrm{h}}\right)\left(1-\mathrm{I}_{\mathrm{m} 1}^{*}\right)\left(\frac{\gamma_{\mathrm{ml} \rightarrow \mathrm{h}} \mathrm{A}_{1} \mathrm{~S}_{\mathrm{h}}^{*}}{\mu_{\mathrm{m} 1}}\right)-\left(\mu_{\mathrm{h}}+\frac{\gamma_{\mathrm{ml} \rightarrow \mathrm{h}} \mathrm{A}_{1} \mathrm{I}_{\mathrm{m} 1}^{*}}{\mu_{\mathrm{m} 1}}+\frac{\gamma_{\mathrm{m} 2 \rightarrow \mathrm{h}} \mathrm{A}_{2} \mathrm{I}_{\mathrm{m} 2}^{*}}{\mu_{\mathrm{m} 2}}\right) \times \\
& \left\{-\left(\mu_{\mathrm{m} 2}+\gamma_{\mathrm{h}} \mathrm{N}_{\mathrm{h}} \mathrm{I}_{\mathrm{h}}^{*}\right)\left(\mu_{\mathrm{m} 1}+\mathrm{r}_{\mathrm{h}}+\mu_{\mathrm{h}}+\gamma_{\mathrm{h}} \mathrm{N}_{\mathrm{h}} \mathrm{I}_{\mathrm{h}}^{*}\right)-\left(\mathrm{r}_{\mathrm{h}}+\mu_{\mathrm{h}}\right)\left(\mu_{\mathrm{m} 1}+\gamma_{\mathrm{h}} \mathrm{N}_{\mathrm{h}} \mathrm{I}_{\mathrm{h}}^{*}\right)+\left(\gamma_{\mathrm{h}} \mathrm{N}_{\mathrm{h}}\right)\left[\left(1-\mathrm{I}_{\mathrm{m} 2}^{*}\right)\left(\frac{\gamma_{\mathrm{m} 2 \rightarrow \mathrm{h}} \mathrm{A}_{2} \mathrm{~S}_{\mathrm{h}}^{*}}{\mu_{\mathrm{m} 2}}\right)+\left(1-\mathrm{I}_{\mathrm{m} 1}^{*}\right)\left(\frac{\gamma_{\mathrm{m} 1 \rightarrow \mathrm{h}} \mathrm{A}_{1} \mathrm{~S}_{\mathrm{h}}^{*}}{\mu_{\mathrm{m} 1}}\right)\right]\right\} \\
& -\left(\frac{\gamma_{\mathrm{m} 1 \rightarrow \mathrm{h}} \mathrm{A}_{1} \mathrm{I}_{\mathrm{m} 1}^{*}}{\mu_{\mathrm{m} 1}}+\frac{\gamma_{\mathrm{m} 2 \rightarrow \mathrm{h}} \mathrm{A}_{2} \mathrm{I}_{\mathrm{m} 2}^{*}}{\mu_{\mathrm{m} 2}}\right)\left(\gamma_{\mathrm{h}} \mathrm{N}_{\mathrm{h}}\right)\left[\left(1-\mathrm{I}_{\mathrm{m} 2}^{*}\right)\left(\frac{\gamma_{\mathrm{m} 2 \rightarrow \mathrm{h}} \mathrm{A}_{1} \mathrm{~S}_{\mathrm{h}}^{*}}{\mu_{\mathrm{m} 2}}\right)+\left(1-\mathrm{I}_{\mathrm{m} 1}^{*}\right)\left(\frac{\gamma_{\mathrm{m} 1 \rightarrow \mathrm{h}} \mathrm{A}_{1} \mathrm{~S}_{\mathrm{h}}^{*}}{\mu_{\mathrm{m} 1}}\right)\right] \\
& \mathrm{b}_{4}=-\left(\mu_{\mathrm{h}}+\frac{\gamma_{\mathrm{m} 1 \rightarrow \mathrm{h}} \mathrm{A}_{1} \mathrm{I}_{\mathrm{m} 1}^{*}}{\mu_{\mathrm{m} 1}}+\frac{\gamma_{\mathrm{m} 2 \rightarrow \mathrm{h}} \mathrm{A}_{2} \mathrm{I}_{\mathrm{m} 2}^{*}}{\mu_{\mathrm{m} 2}}\right) \\
& \left\{-\left(r_{h}+\mu_{h}\right)\left(\mu_{m 1}+\gamma_{h} N_{h} I_{h}^{*}\right)\left(\mu_{m 2}+\gamma_{h} N_{h} I_{h}^{*}\right)+\gamma_{h} N_{h} S_{h}^{*}\left[\left(1-I_{m 1}^{*}\right)\left(\mu_{m 2}+\gamma_{h} N_{h} I_{h}^{*}\right)\left(\frac{\gamma_{m 1 \rightarrow h} A_{1}}{\mu_{m 1}}\right)+\left(1-I_{m 2}^{*}\right)\left(\mu_{m 1}+\gamma_{h} N_{h} I_{h}^{*}\right)\left(\frac{\gamma_{m 2 \rightarrow h} A_{2}}{\mu_{m} 2}\right)\right]\right\} \\
& -\left(\frac{\gamma_{\mathrm{m} 1 \rightarrow \mathrm{h}} \mathrm{A}_{1} \mathrm{I}_{\mathrm{m} 1}^{*}}{\mu_{\mathrm{m} 1}}+\frac{\gamma_{\mathrm{m} 2 \rightarrow \mathrm{h}} \mathrm{A}_{2} \mathrm{I}_{\mathrm{m} 2}^{*}}{\mu_{\mathrm{m} 2}}\right)\left(\gamma_{\mathrm{h}} \mathrm{N}_{\mathrm{h}} \mathrm{S}_{\mathrm{h}}^{*}\right)\left[\left(1-\mathrm{I}_{\mathrm{m} 1}^{*}\right)\left(\mu_{\mathrm{m} 2}+\gamma_{\mathrm{h}} \mathrm{N}_{\mathrm{h}} \mathrm{I}_{\mathrm{h}}^{*}\right)\left(\frac{\gamma_{\mathrm{m} 1 \rightarrow \mathrm{h}} \mathrm{A}_{1}}{\mu_{\mathrm{m} 1}}\right)+\left(1-\mathrm{I}_{\mathrm{m} 2}^{*}\right)\left(\mu_{\mathrm{m} 1}+\gamma_{\mathrm{h}} \mathrm{N}_{\mathrm{h}} \mathrm{I}_{\mathrm{h}}^{*}\right)\left(\frac{\gamma_{\mathrm{m} 2 \rightarrow \mathrm{h}} \mathrm{A}_{2}}{\mu_{\mathrm{m} 2}}\right)\right]
\end{aligned}
$$


The four eigenvalues of $\lambda^{4}+b_{1} \lambda^{3}+b_{2} \lambda^{2}+b_{3} \lambda+b_{4}=0$ will have negative real part if they satisfy the RouthHurwitz criteria. Thus, $\mathrm{E}_{1}$ will be locally asymptotically stable for $\mathrm{R}_{0}>1$ when:

$$
\begin{aligned}
& b_{1}>0 \\
& b_{3}>0 \\
& b_{4}>0 \\
& b_{1} b_{2} b_{3}>b_{3}^{2}+b_{1}^{2} b_{4}
\end{aligned}
$$

$\lambda^{4}+b_{1} \lambda^{3}+b_{2} \lambda^{2}+b_{3} \lambda+b_{4}=0$

Satisfies the following conditions Equation 20:

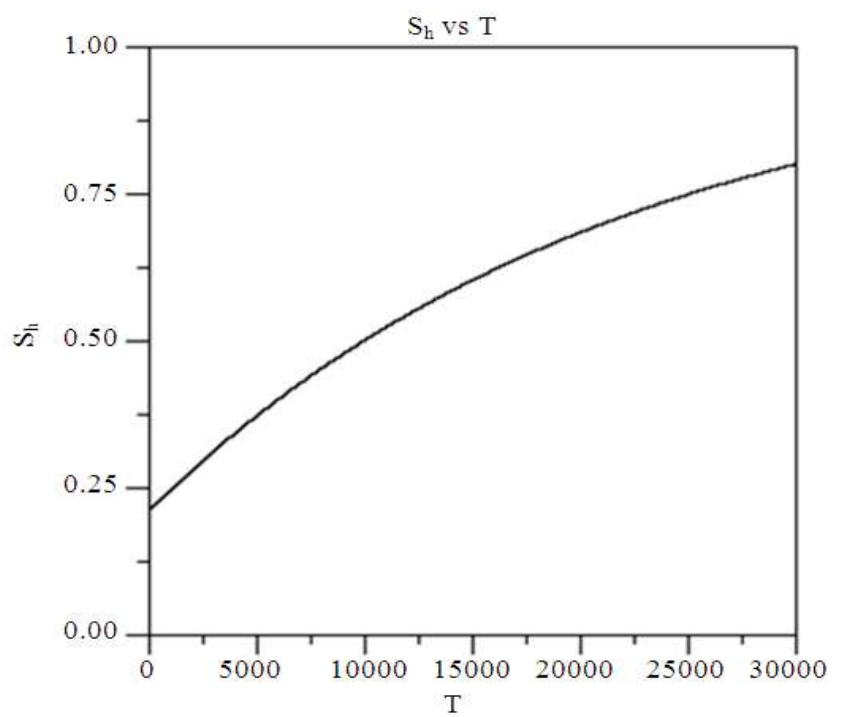

(a)

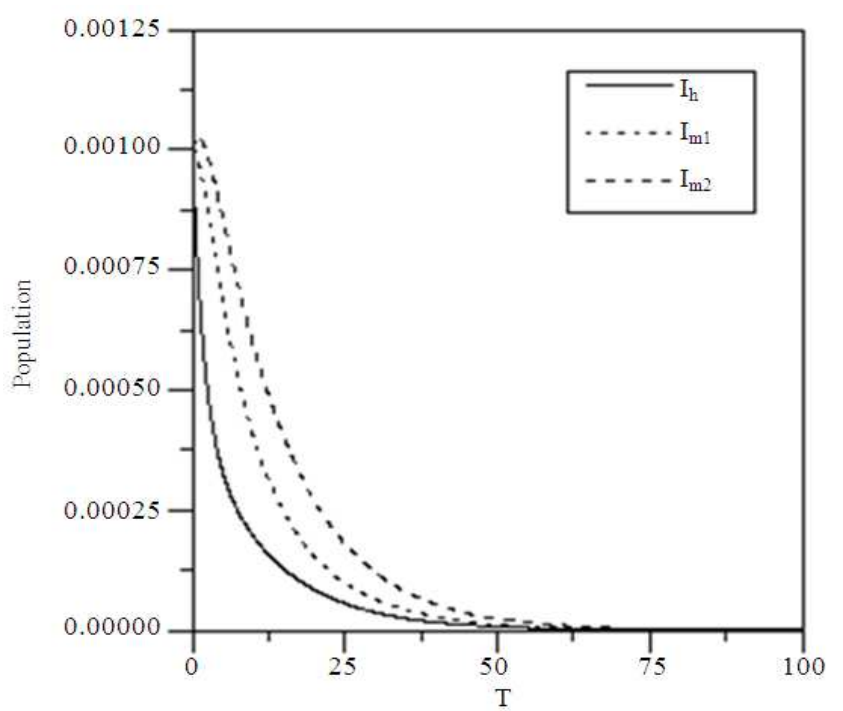

(b)
The parameters used in the numerical simulation are given in Table 1.

\section{RESULTS}




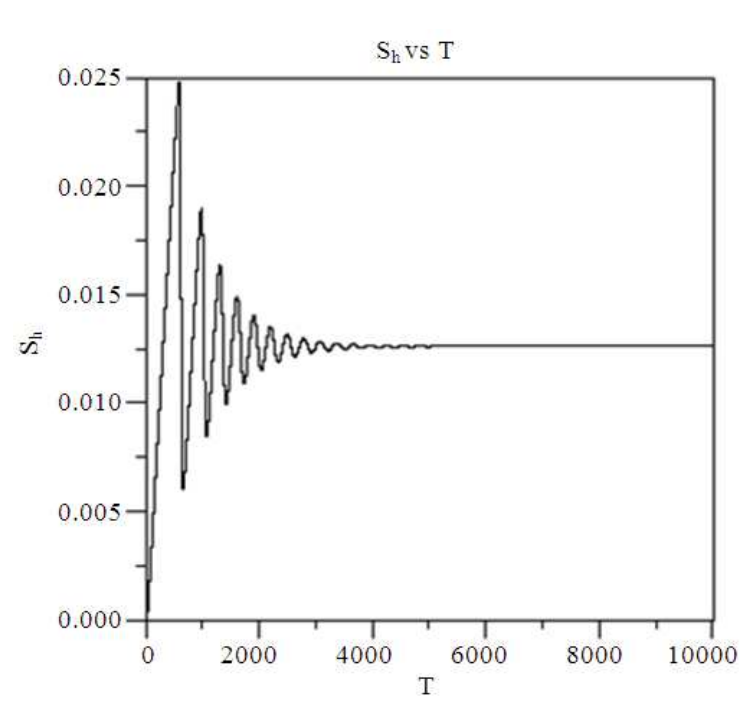

(a)

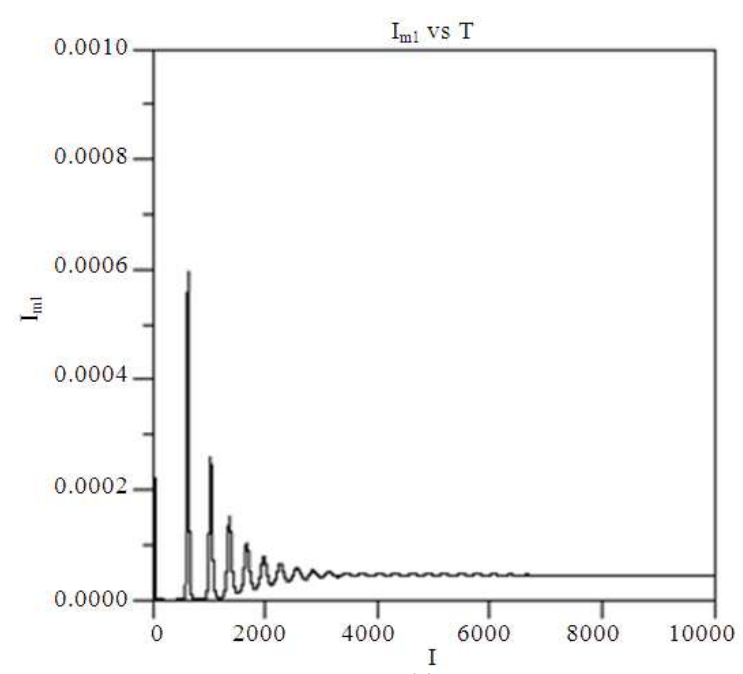

(c)

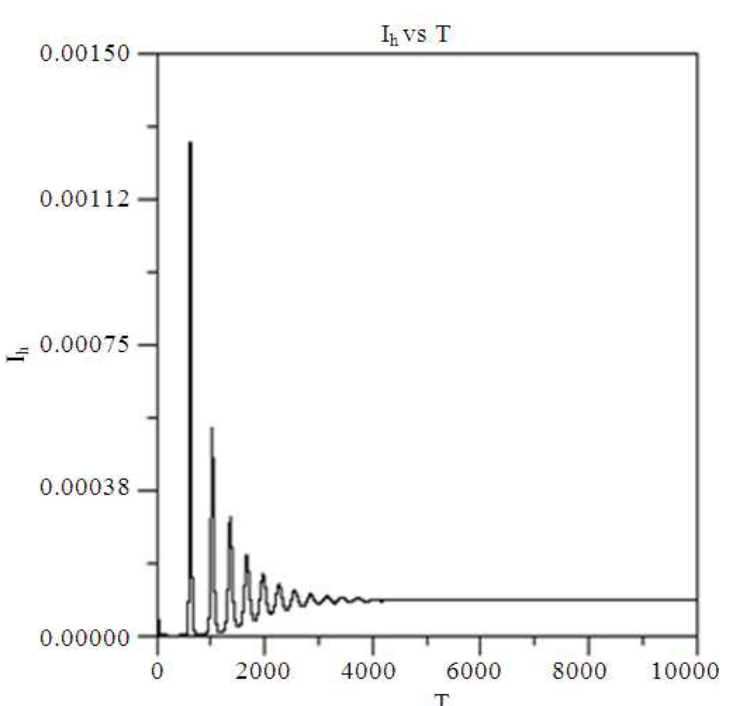

(b)

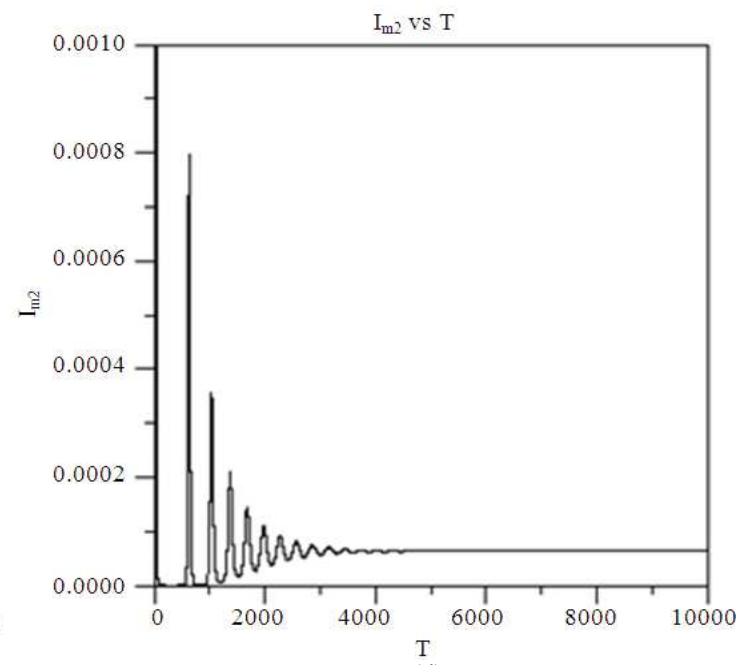

(d)

Fig. 3. Time series of (a) susceptible human, (b) infected human, (c) infected Ae. aegypti mosquito and (d) infected Ae. albopictus mosquito. The values of parameters are in the text, the solutions converge to the endemic equilibrium state $\mathrm{E}_{1}$ as shown

Table 1. Parameter values used in numerical simulations at disease free state

\begin{tabular}{lll}
\hline Parameters & Descriptions & Values \\
\hline$\mu_{\mathrm{h}}$ & Birth (death) rate of human & $1 /(65 \times 365)$ day $^{-1}$ \\
$\mathrm{~N}_{\mathrm{h}}$ & Total number of human population & 20000 \\
$\mathrm{~A}_{1}$ & Recruitment rate of Ae. aegypti mosquito & $90 \mathrm{day}^{-1}$ \\
$\mathrm{~A}_{2}$ & Recruitment rate of Ae. albopictus mosquito & $100 \mathrm{day}^{-1}$ \\
$\gamma_{\mathrm{m} 1 \rightarrow \mathrm{h}}$ & Transmission rate of CHIKV from Ae. aegypti to human & $0.0002 \mathrm{day}^{-1}$ \\
$\gamma_{\mathrm{m} 2 \rightarrow \mathrm{h}}$ & Transmission rate of CHIKV from Ae. albopictus to human & $0.003 \mathrm{day}^{-1}$ \\
$\gamma_{\mathrm{h}}$ & Transmission rate of CHIKV from human to mosquito & $0.00001 \mathrm{day}^{-1}$ \\
$\mathrm{r}_{\mathrm{h}}$ & Recovery rate of human & $1 / 2 \mathrm{day}^{-1}$ \\
$\mu_{\mathrm{m} 1}$ & Death rate of Ae aegypti mosquito & $1 / 5 \mathrm{day}^{-1}$ \\
$\mu_{\mathrm{m} 2}$ & Death rate of Ae albopictus mosquito & $1 / 7 \mathrm{day}^{-1}$ \\
$\mathrm{~b}$ & Biting rate of mosquito & $1 \mathrm{day}^{-1}$ \\
\hline
\end{tabular}




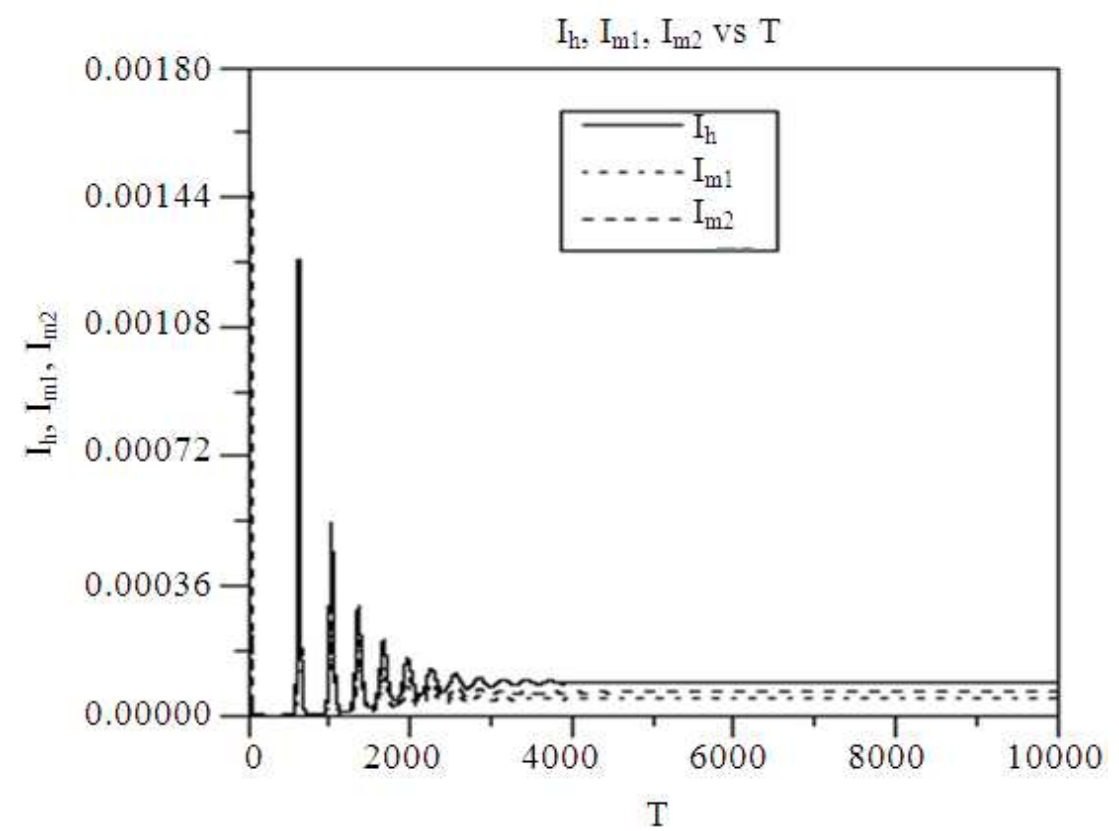

(a)

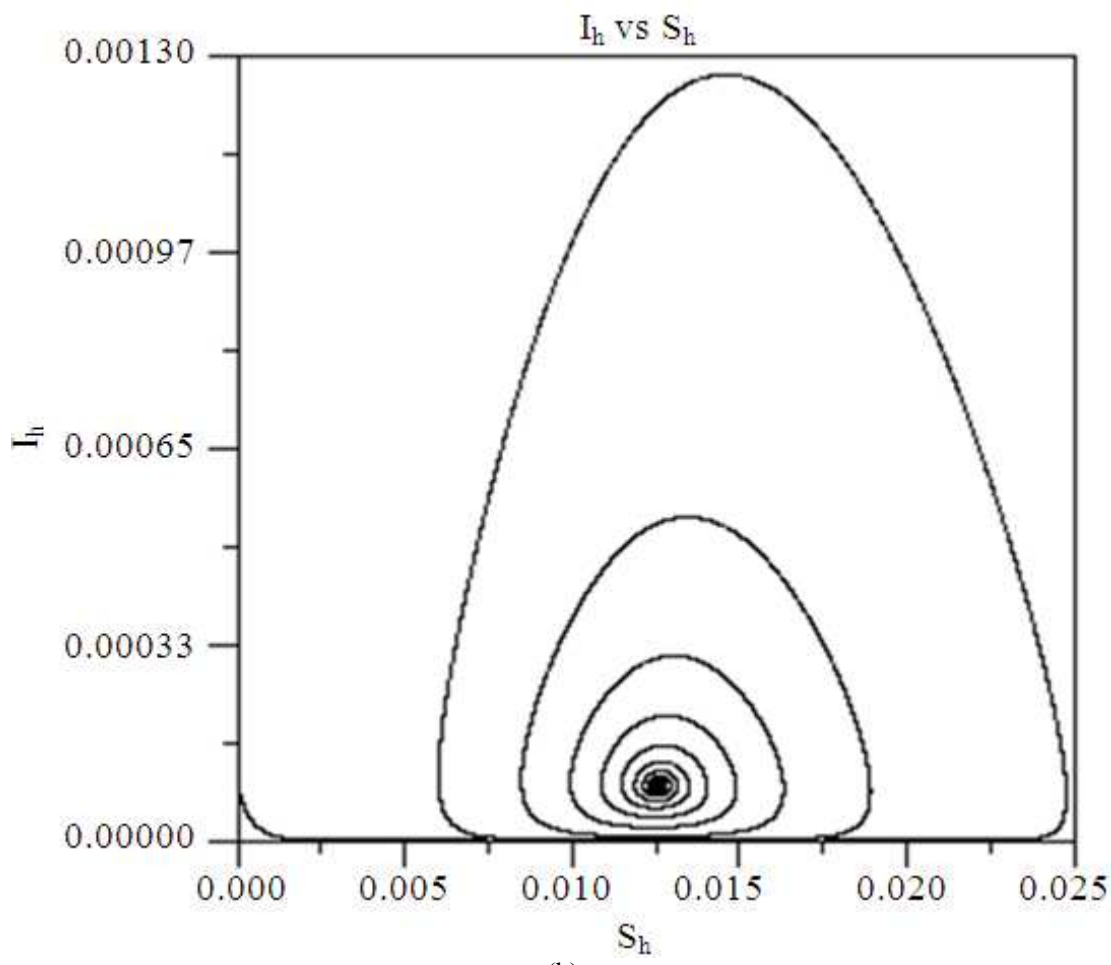

(b)

Fig. 4. (a) Time series of infected human, infected Ae. aegypti mosquito and infected Ae. albopictus mosquito, proportions. (b) Phase portrait of $\left(\mathrm{S}_{\mathrm{h}}-\mathrm{I}_{\mathrm{h}}\right)$ plan with parameters as given in text, the trajectory approach to the endemic state $\mathrm{E}_{1}$ as shown 


\subsection{Stability of Disease Free State}

Using the values of parameters listed in Table 1, we find the eigenvalues and basic reproductive number to be:

$$
\begin{aligned}
& \lambda_{1}=-0.628528, \lambda_{2}=-0.186016, \lambda_{3}=-0.0283587, \\
& \lambda_{4}=-0.00457, R_{0}=0.000548521<1
\end{aligned}
$$

Since all of these eigenvalues are to be negative and the basic reproductive number is less than one, the equilibrium state will be the disease free state, $\mathrm{E}_{0}$ as is shown in Fig. 2.

\subsection{Stability of Endemic State}

We change the value of the recruitment rate of mosquito to $A_{1}=5,000, A_{2}=10,000$ and keep the other values of parameters to be those given in Table $\mathbf{1}$, we find the eigenvalues to be $\lambda_{1}=-0.6285525, \lambda_{2}=$ $0.186016, \lambda_{3}=-0.0283579, \lambda_{4}=-0.004557$ and the basic reproductive number to be $\mathrm{R}_{0}=4571.01>1$. Since all of these eigenvalues are to be negative and the basic reproductive number to be greater than one, the equilibrium endemic state will be locally asymptotically stable $E_{1}$ as seen in Fig. 3.

\section{DISSCUSSION}

We formulated the transmission model of Chikungunya fever by taking into account the presence of two species of Aedes mosquitoes. We found two equilibrium points: Disease free state and endemic disease state. In disease free state, it will be local stability when $\mathrm{R}_{0}<1$ and the endemic disease state will be local stability when $\mathrm{R}_{0}>1$. The basic reproductive number is:

$$
\mathrm{R}_{\mathrm{o}}=\mathrm{R}_{01}+\mathrm{R}_{02}=\frac{\gamma_{\mathrm{h}} \mathrm{N}_{\mathrm{h}} \gamma_{\mathrm{ml} \rightarrow \mathrm{h}} \mathrm{A}_{1}}{\left(\mathrm{r}_{\mathrm{h}}+\mu_{\mathrm{h}}\right)\left(\mu_{\mathrm{m} 1} \mu_{\mathrm{m} 1}\right)}+\frac{\gamma_{\mathrm{h}} \mathrm{N}_{\mathrm{h}} \gamma_{\mathrm{m} 2 \rightarrow \mathrm{h}} \mathrm{A}_{2}}{\left(\mathrm{r}_{\mathrm{h}}+\mu_{\mathrm{h}}\right)\left(\mu_{\mathrm{m} 2} \mu_{\mathrm{m} 2}\right)}
$$

It represents the number of secondary case that one case can produce.

\section{CONCLUSION}

In this study, we have analyzed a transmission model for Chikungunya fever in which two species of mosquitoes are present. This is important to the modeling of this disease in Thailand since both the $A e$. aegypti and Ae. albopictus mosquitoes are present. In most other countries, only one specie of the Aedes mosquito is present. Again in Thailand, it was found that both species can inhabit the same breeding sites. By reducing the number of the breeding sites, the recruitment rates of both Aedes mosquitoes would be reduced. From Fig. 2, changing the recruitment rate $A_{1}$ and $A_{2}$ to 5,000 and 10,000, respectively, we find that the time behaviors of the infected human, the Ae. aegypti and the Ae. albopictus mosquitoes decrease sinsodially to the endemic state (Fig. 4a). Figure 4b shows the trajectory of the human population in the $\mathrm{S}_{\mathrm{h}}-\mathrm{I}_{\mathrm{h}}$ phase space. The changes seen in the time behaviors shown in Fig. 3 and 4 show that the destruction of the breeding cause equilibrium state to change from being an endemic equilibrium state to a disease free equilibrium state.

\section{ACKNOWLEDGEMENT}

Surapol Naowarat would like to thank Suratthani Rajabhat University for financial support according to Code: 2554A1590213.

\section{REFERENCES}

Allen, L.J.S., 2006. An Introduction to Mathematical Biology. Pearson/Prentice Hall, Upper Saddle River, New Jersey, ISBN-10: 0130352160, pp: 348.

Driessche, P.V.D. and J. Watmough, 2002. Reproduction numbers and sub-threshold endemic equilibria for compartmental models of disease transmission. Math. Biosci., 180: 29-48. PMID: 12387915

Kucharz, E.J. and L. Cebula-Byrska, 2012. Chikungunya fever. Eur. J. Int. Med., 23: 325-329. DOI: 10.1016/j.ejim.2012.01.009

Moulay, D. and Y. Pigne, 2013. A metapopulation model for Chikungunya including populations mobility on a large-scale network. J. Theor. Biol., 318: 129-139. DOI: $10.1016 /$ j.jtbi.2012.11.008

Moulay, D., M.A. Aziz-Alaoui and M. Cadivel, 2011. The chikungunya disease: Modeling, vector and transmission global dynamics. Math. Biosci., 229: 50-63. PMID: 21070789

PAHO, 2011. Preparedness and response for Chikungunya virus: Introduction in the Americas. Washington, D.C.

Poletti, P., G. Messeri M. Ajelli, R. Vallorni. and C. Rizzo et al., 2011. Transmission potential of Chikungunya virus and control measures: The case of Italy. PlOS One, 6: e18860-e18860. DOI: 10.1371/journal.pone.0018860 
Pongsumpun, P., 2010. Dynamical transmission model of Chikungunya in Thailand. World Acad. Sci. Eng. Technol., 68: 1161-1165.

Preechaporn, W., M. Jaroensutasinee and K. Jaroensutasinee, 2007. Seasonal prevalence of Aedes aegypti and Ae. albopictus in three topographical areas of southern Thailand. World Acad. Sci. Eng. Technol., 12: 23-27.

Tilston, N., C. Skelly and P. Weinstein, 2009. PanEuropean Chikungunya surveillance: Designing risk stratified surveillance zones. Int. J. Health Geogr., 8: 61-61. DOI: 10.1186/1470-072X-8-61
Tsetsarkin, K.A., 2009. A single mutation in Chikungunya virus affects vector specificity and epidemic potential. PIOS Pathong., 3: e201-e201. PMID: 18069894

Yakob, L. and A.C.A. Clements, 2013. A mathematical model of Chikungunya dynamics and control: The major epidemic on Reunion Island. PLOS One, 8: e57448-e57448. DOI: 